\title{
Composantes génétiques des paramètres de croissance dans le croisement entre les races Aberdeen Angus et Charolaise
}

\author{
Maria Cristina MIQUEL et H.A. MOLINUEVO \\ Instituto Nacional de Tecnologia Agropecuaria, \\ Estación Experimental Regional Agropecuaria de Balcarce, \\ Balcarce, Provincia de Buenos-Aires Argentina
}

\begin{abstract}
Résumé
Cette étude présente des estimations des effets d'hétérosis direct et maternel entre les races Aberdeen Angus et Charolaise, ainsi que des différences entre ces races pour les effets génétiques directs et maternels et les pertes des effets d'interaction entre allèles lors des recombinaisons chromosomiques. Les analyses ont concerné les poids à la naissance, au sevrage et à 20 mois, et la croissance absolue et relative entre ces différents poids. 815 veaux nés entre 1966 et 1971 à la «Estación Experimental Agropecuaria - I.N.T.A.» de Balcarce (Argentine) ont été ainsi analysés de la naissance au sevrage. Pour la phase après sevrage, 605 animaux seulement ont servi à cette étude. Les animaux étaient soit de races pures : Aberdeen Angus ou Charolaise, soit croisés entre ces deux races : F1, F2 et croisements de retour. Les effets directs de la race Charolaise sont significativement supérieurs à ceux de la race Aberdeen Angus pour tous les caractères. Les effets maternels de la race Charolaise ne sont supérieurs à ceux de l'Aberdeen Angus que pour le poids à la naissance. Les effets d'hétérosis directs sont significativement différents de zéro pour le poids à la naissance $(11 \mathrm{p} .100)$ et le poids à 20 mois $(12$ p. 100). Les effets d'hétérosis maternels atteignent des valeurs maximum pour la phase naissance-sevrage $(7 \mathrm{p} .100)$ mais ne s'avèrent dans aucun cas statistiquement significatifs. La perte d'effets épistatiques due aux recombinaisons chromosomiques n'apparaît importante pour aucun caractère.
\end{abstract}

\section{I. - Introduction}

En 1958 l'Institut National de Technologie Agraire ( Instituto Nacional de Tecnologia Agropecuaria» - I.N.T.A.) a introduit la race Charolaise dans ses programmes expérimentaux, en vue d'accroître la productivité et notamment les aptitudes bouchères des bovins à viande en Argentine. Des taureaux de race Charolaise et de la semence ont été ainsi utilisés pour féconder des vaches des races britanniques traditionnelles dans le pays. Lopez Saubidet et al. (1963) et Cabrini et Cavandoli (1964) ont fait connaître les premiers résultats obtenus par ces croisements sur les aptitudes bouchères. Suite à cette évaluation, nous avons comparé ces divers croisements sur les performances de reproduction des femelles croisées F1 (Charolaise $\times$ Aberdeen Angus) et sur leurs aptitudes maternelles (croissance avant et après sevrage et caractéristiques de la viande, des produits issus de ces femelles). C'est ainsi que Molinuevo 
et al. (1971) ont réalisé une étude sur les performances de reproduction de ces mères croisées. L'objet de notre étude est d'analyser les performances de croissance avant et après sevrage des veaux engendrés par ces divers types de mères, qui sont soit de races pures (Charolaise ou Aberdeen Angus), soit croisés (F1, F2 et croisements de retour).

Cette analyse a pour but d'estimer à partir de ces données, les différentes composantes génétiques de la croissance lors du croisement entre les races Charolaise et Aberdeen Angus c'est-à-dire les effets directs et maternels sur l'hétérosis et la valeur génétique additive ainsi que les effets d'interaction dus aux recombinaisons chromosomiques en croisement.

\section{II. - Matériel et méthode}

Les 815 veaux utilisés pour évaluer la croissance avant sevrage, sont nés à la Station expérimentale (Estación Experimental Regional Agropecuaria) de l'I.N.T.A. à Balcace entre 1966 et 1971. La conduite de ces animaux et les conditions de réalisation de l'expérimentation ont été décrites lors des études antérieures (MoLinuEvo, 1970 ; Molinuevo et al., 1971 ; Departamento de Producción animal, 1974).

Ces veaux ont été procréés par insémination artificielle entre la mi-octobre et la mi-janvier. 6 taureaux par an et par type génétique ont été utilisés, parmi lesquels de un à trois d'entre eux au moins étaient réutilisés l'année suivante. Pour la race Charolaise, la semence a été importée de France.

Les veaux mâles ont été castrés vers l'âge de 2 mois. Tous les veaux ont été sevrés à l'âge de 200 jours plus ou moins une semaine. Ensuite, ils ont été transportés dans la région Ouest de la Province de Buenos Aires pour être engraissés à l'herbe sur des prairies temporaires jusqu'à l'âge d'un an et demi environ; l'abattage des bouvillons n'intervenant qu'au-delà du poids de $400 \mathrm{~kg}$. Seuls les animaux nés entre 1965 et 1970 ont servi à l'analyse de cette phase après sevrage, soit 604 animaux.

Les veaux ont été pesés à la naissance, c'est-à-dire durant leur première semaine de vie, puis tous les 28 jours avant et après le sevrage. Le gain de poids journalier absolu a été calculé comme étant la régression du poids sur l'âge, et le gain de poids journalier relatif comme étant la régression du logarithme naturel du poids sur l'âge. Pour ces gains de poids relatifs, les résultats ont été multipliés par 100. Les gains de poids jusqu'au sevrage ont été estimés à partir des pesées effectuées de la naissance au sevrage pour la croissance avant sevrage, et à partir des pesées réalisées du sevrage jusqu'au début mars de la seconde année de vie de l'animal (c'est-à-dire entre l'âge de 19 et 21 mois) pour la croissance après sevrage. Le poids au sevrage a été ajusté à l'âge de 210 jours à partir de la croissance calculée avant sevrage et du poids de naissance. De même, le poids à 20 mois a été obtenu à partir du poids au sevrage ajusté à 210 jours et du gain de poids calculé après sevrage.

Ces performances ont été analysées en vue d'obtenir les estimations des effets moyens relatifs à chacun des types génétiques des animaux considérés (tableau 1), à partir d'une analyse par moindres-carrés (SEARLE, 1971). Le modèle d'analyse des performances avant sevrage (avec effets fixés) incluait les effets du type génétique, 
du sexe, de l'année et du mois de naissance du veau ainsi que l'effet du rang de vêlage de la mère. Pour les performances après sevrage, l'effet du rang de vêlage des mères a été exclu du modèle.

\section{TABLEAU 1}

Schéma des différents types d'accouplements réalisés et nomenclature des types génétiques des produits.

Mating design and abbreviations used for genetic types of offspring.

\begin{tabular}{|c|c|c|c|}
\hline \multirow[b]{2}{*}{ Mère (dam) } & \multicolumn{3}{|c|}{ Père (sire) } \\
\hline & $\begin{array}{l}\text { Aberdeen Angus } \\
\text { (A) }\end{array}$ & $\begin{array}{l}\text { Charolais } \\
\text { (C) }\end{array}$ & $\begin{array}{c}\mathbf{F}_{1} \\
(\mathrm{CA})\end{array}$ \\
\hline $\begin{array}{l}\text { Aberdeen Angus (A) } \ldots \ldots \ldots \ldots \ldots \ldots \\
\text { Charolaise }(\mathrm{C}) \ldots \ldots \ldots \ldots \ldots \ldots \ldots \\
\mathrm{F}_{1}(\mathrm{C} \times \mathrm{A}) \ldots \ldots \ldots \ldots\end{array}$ & $\frac{\mathrm{AA}}{\overline{\mathrm{A}}(\mathrm{CA})}$ & $\begin{array}{c}\text { CA } \\
\mathrm{CC} \\
\mathrm{C}(\mathrm{CA})\end{array}$ & $\begin{array}{c}(\mathrm{CA}) \mathrm{A} \\
(\mathrm{CA})(\mathrm{CA})\end{array}$ \\
\hline
\end{tabular}

N.B. : $\mathrm{C} \times \mathrm{A}=$ croisement d'un père de type génétique $\mathrm{C}$ avec une mère de type génétique $\mathrm{A}$.

A partir des effets du type génétique des veaux ainsi obtenus, nous avons estimé les différentes composantes génétiques dues au croisement entre les deux races Charolaise et Aberdeen Angus, c'est-à-dire les effets génétiques additifs directs ( $\mathrm{gI}$ ) et maternels $\left(\mathrm{g}^{\mathrm{M}}\right)$, les effets d'hétérosis directs $\left(\mathrm{h}^{\mathrm{I}}\right)$ et maternels $\left(\mathrm{h}^{\mathrm{M}}\right)$, ainsi que les pertes de recombinaisons épistatiques $\left(\mathrm{r}^{\mathrm{I}}\right)$. Pour cela nous avons décomposé chaque type génétique selon l'espérance de ces paramètres génétiques définis par Dickerson (1969), d'après la méthode décrite par Foulley et LEFORT (1978) pour ce cas particulier. Toutefois, du fait que nous employons la terminologie décrite par Dickerson (1969), les effets de recombinaisons exprimés dans cette étude représentent la moitié de ceux définis par FOULLEY et LEFORT (1978) qui se réfèrent à la définition donnée par DiCKERSON (1972).

\section{III. - Résultats et discussion}

Pour chaque caractère analysé dans le tableau 2 figurent les estimées des effets moyens et leurs écarts-types par type génétique, ainsi que le nombre d'observations correspondant. Les différents paramètres génétiques pour ces mêmes caractères sont rassemblés dans le tableau 3 :

\section{Effets génétiques additifs directs $\left(g^{I}\right)$}

La race Charolaise possède des effets additifs directs significativement supérieurs à ceux de la race Aberdeen Angus pour le poids à la naissance, le poids au sevrage 


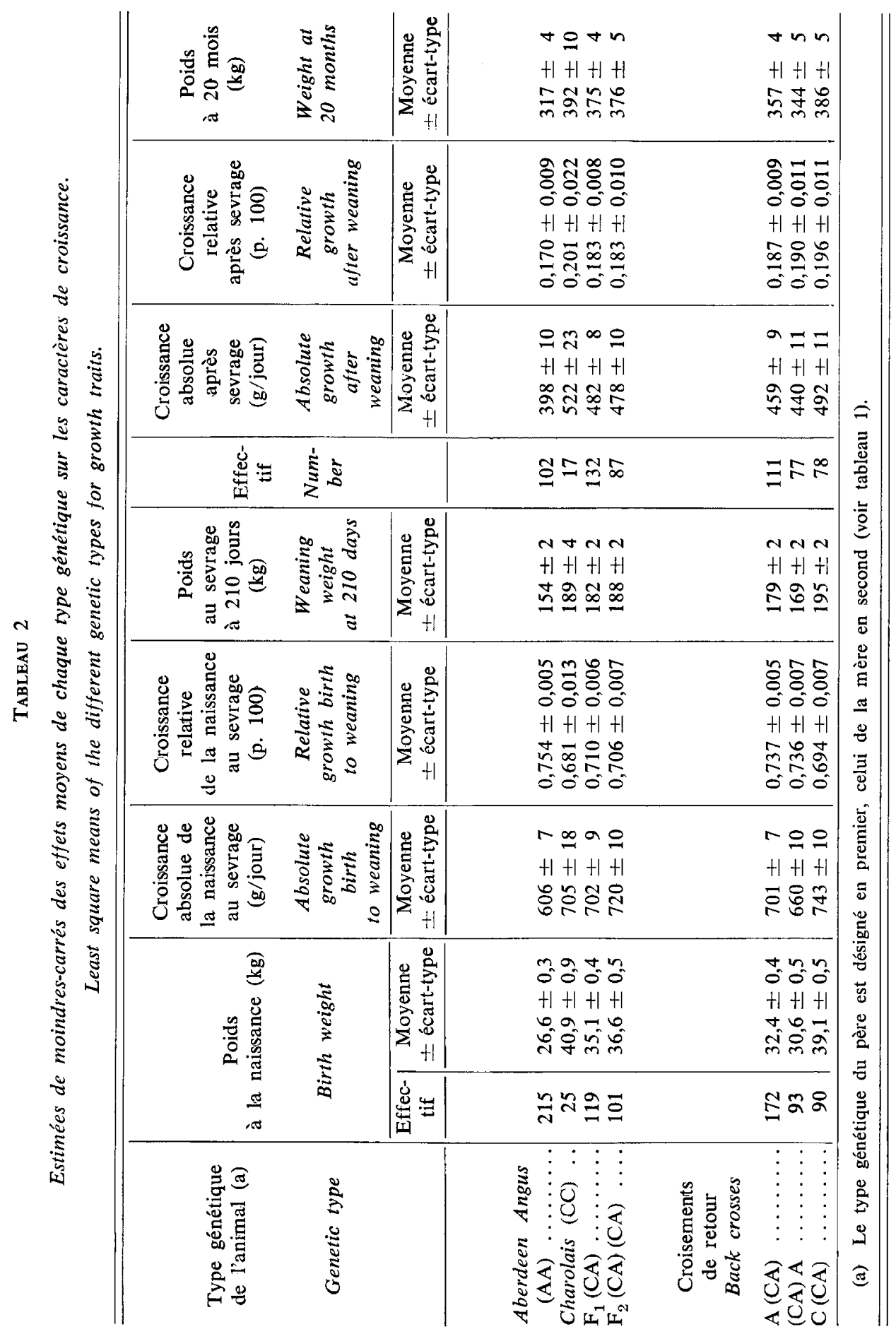


et le poids à 20 mois ainsi que pour la croissance journalière absolue entre ces différents poids (tableau 3). Cette supériorité d'effets directs de la race Charolaise sur l'Aberdeen Angus pour les caractères de croissance absolue avant et après le sevrage, est déjà relativement bien connue (JAIN et al., 1971; LASLEY et al., 1973 ; Miquel et al., 1974 a et b ; Sagebiel et al., 1974 ; Peacock et al., 1978).

A la naissance les effets directs de la race Charolaise sont de 20 p. 100 supérieurs à ceux de la race Aberdeen Angus, alors qu'au sevrage à 210 jours cette supériorité n'atteint plus que 9 p. 100 . Cette réduction de la différence entre la naissance et le sevrage résulte d'une meilleure croissance relative de la race Aberdeen Angus durant cette phase. Cette supériorité de croissance relative de la race Aberdeen Angus sur la race Charolaise a d'ailleurs déjà été observée par Molinuevo (1967) en comparant les poids métaboliques de veaux Aberdeen Angus et Charolais aux âges de 40 et de 222 jours. La différence de croissance relative avant sevrage entre ces deux races pour les effets additifs directs, est également à rapprocher de l'existence d'une variabilité génétique intra race de ces effets directs observée par GREGORY et al. (1978) en race Angus, Hereford, Red Poll et Brown Swiss. Par ailleurs, après sevrage, les effets directs des deux races comparées sont identiques pour la croissance relative (tableau 3).

\section{Effets génétiques additifs maternels $\left(g^{M}\right)$}

La race Charolaise présente également un effet maternel significativement supérieur ( +7 p. 100) à celui de la race Aberdeen Angus sur le poids à la naissance des veaux (tableau 3). Par contre il n'y a pas de différence sur la croissance absolue jusqu'au sevrage et sur le poids au sevrage, alors que la race Aberdeen Angus a un effet maternel supérieur $(+3,0$ p. 100) mais non significatif à celui de la race Charolaise sur la croissance relative jusqu'au sevrage. Ces résultats ne concordent pas très bien avec ceux de SAgebiel et al. (1974) et PeAcock et al. (1978) qui trouvent qu'entre les croisements réciproques Charolais $\times$ Angus, les veaux issus des mères Charolaises ont une meilleure croissance avant sevrage que ceux nés de mère Angus. Le Charolais utilisé par ces auteurs, d'origine américaine, provient de croisements d'absorption, alors que celui utilisé dans notre étude est importé de France; la différence d'origine du matériel génétique pourrait être en partie responsable des différences de comportement observées sur ces effets maternels. Les différences de conduite des animaux entre ces expériences pourraient aussi expliquer une partie de ces divergences.

Les effets maternels des races Aberdeen Angus et Charolaise ne diffèrent pas significativement pour la croissance après sevrage et pour le poids à 20 mois (tableau 3), bien que les produits des mères Charolaises aient une croissance absolue après sevrage et un poids à 20 mois supérieurs de +7 p. 100 et +6 p. 100 respectivement par rapport aux produits des mères Aberdeen Angus. Il serait logique d'observer que les produits de la race maternelle à plus faibles effets maternels sur la croissance avant sevrage, manifestent une meilleure croissance (croissance compensatrice) après sevrage conduisant ainsi à trouver des effets maternels supérieurs sur la croissance après sevrage pour cette race. Toutefois, ceci ne peut se manifester pleinement qu'avec un système d'engraissement intensif que les conditions de pâturages dans lesquelles s'est déroulée notre expérience ne permettent pas toujours. 


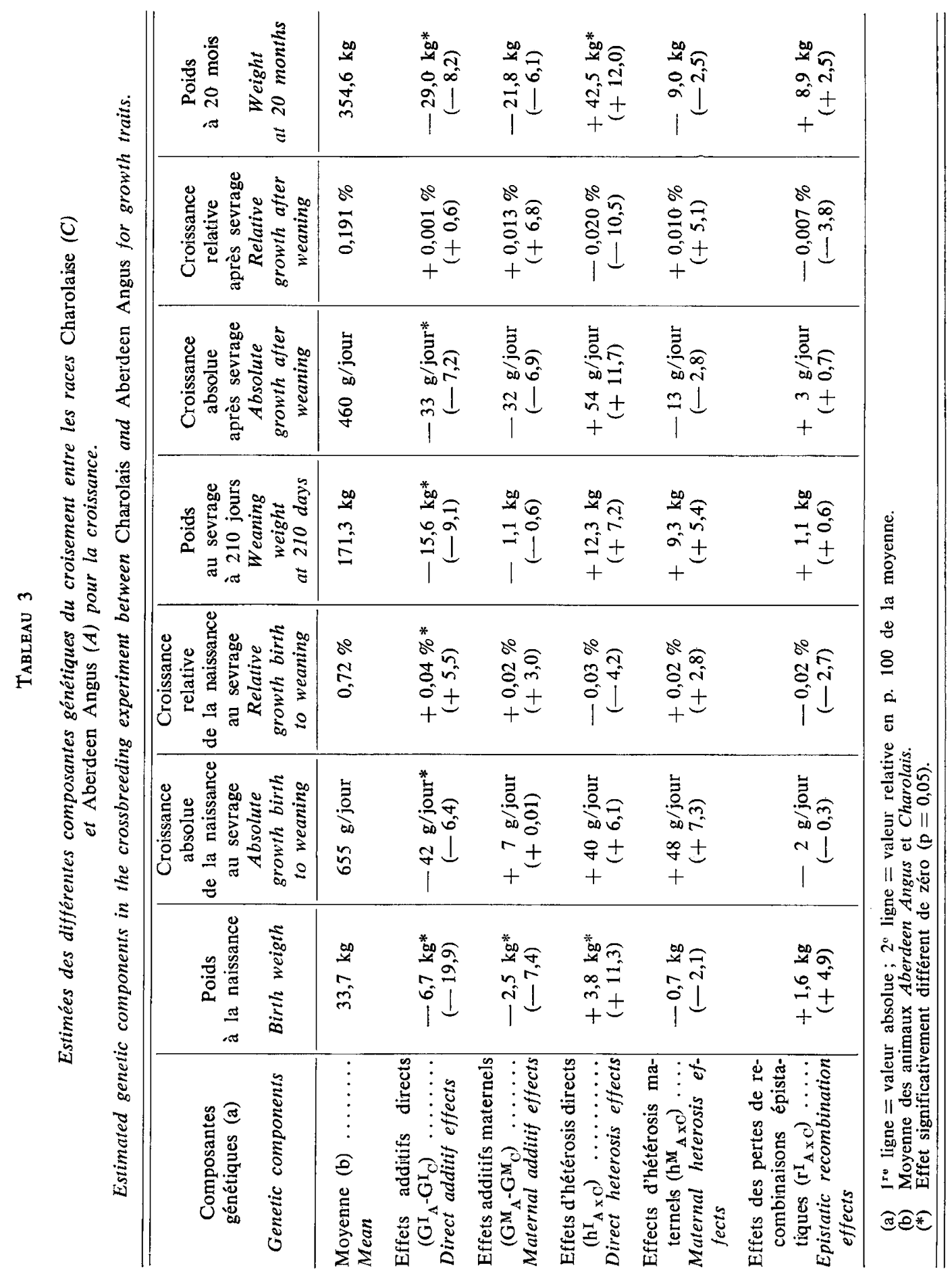




\section{Effets d'hétérosis direct $\left(h^{I}\right)$}

Seuls les effets d'hétérosis direct sur le poids à la naissance et le poids à 20 mois sont significativement différents de zéro : +11 p. 100 et +12 p. 100 respectivement (tableau 3). Les valeurs d'hétérosis direct sur le poids au sevrage $(+7$ p. 100) et sur la croissance absolue avant $(+6$ p. 100) et après $(+12$ p. 100) sevrage, bien que non significativement différentes de zéro, restent encore importantes notamment pour la croissance absolue après sevrage. Les effets d'hétérosis direct tendent à être négatifs pour la croissance relative avant $(-4$ p. 100) et après $(-11$ p. 100) sevrage.

Les valeurs estimées dans cette étude pour les effets d'hétérosis direct sur les caractères de croissance absolue, sont plutôt supérieures à celles rencontrées dans la bibliographie pour le croisement entre ces deux races : +2 à +5 p. 100 pour la croissance avant sevrage et le poids au sevrage (SAgebiel et al., 1974 ; PeAcock et al., 1978). Utilisant une partie des données de notre étude, Molinuevo (1970) avait déjà trouvé pour le poids à la naissance et au sevrage des valeurs comparables à celles que nous avons estimées. Signalons que dans son étude, Molinuevo (1970) a estimé les effets d'hétérosis direct en utilisant les résultats de mères $F 1$, ce qui fait que ses estimations incluent également les effets de recombinaisons épistatiques. LASL.EY et al. (1973) n'observent pas de différences significatives pour des bouvillons au pâturage entre les Charolais, les Angus et les croisés, contrairement à ce qu'indiquent nos résultats d'hétérosis direct sur le poids à 20 mois. Comme précédemment les différences d'origine du Charolais et de conduite des animaux entre les expériences nord américaines et notre étude, peuvent être à la base de ces divergences de résultats sur les effets d'hétérosis direct.

\section{Effets d'hétérosis maternel $\left(h^{M}\right)$}

Les effets d'hétérosis maternel les plus importants sont ceux concernant la croissance absolue avrant sevrage $(+7 \mathrm{p} .100)$, le poids au sevrage $(+5 \mathrm{p} .100)$ et la croissance relative après sevrage $(+5 \mathrm{p} .100)$, mais aucune de ces valeurs n'est significativement différente de zéro (tableau 3). Pour les autres caractères, tels que le poids à la naissance, la croissance relative avant le sevrage, la croissance absolue après sevrage et le poids à 20 mois, les effets d'hétérosis maternel sont pratiquement nuls (entre +3 et -3 p. 100).

Pour les races britanniques Hereford, Angus et Shorthorn, les effets d'hétérosis maternel estimés par CUNDIFF et al. (1974) sont assez proches de nos estimations $(+2$ p. 100 pour le poids à la naissance et +4 p. 100 pour le poids au sevrage) tout comme les estimations de OLson et al. (1978) pour la croissance après sevrage (effets d'hétérosis maternel pratiquement nuls). Ces résultats indiquent que les effets d'hétérosis maternel sont plus importants pour la croissance avant sevrage que pour la croissance après sevrage, ce qui concorde assez bien avec nos observations.

\section{Effets de recombinaison directe $\left(r^{I}\right)$}

En général, les effets des pertes épistatiques dues aux recombinaisons chromosomiques que nous observons (tableau 3 ) ne sont pas très importants et en aucun cas 
ils ne sont significativement différents de zéro. La valeur la plus élevée est celle concernant le poids à la naissance $(+5 \mathrm{p} .100)$.

Si nous admettons que ces effets sont nuls, cela implique que les effets d'hétérosis dépendent directement de l'hétérozygotie. D'ailleurs dans une analyse préliminaire de données de croisements rotatifs entre Angus, Hereford et Shorthorn, CunDIFF (1978 - données non publiées) obtient des valeurs d'hétérosis proportionnelles à l'hétérozygotie des animaux pour le poids au sevrage et la fertilité de femelles croisées.

\section{IV. - Conclusions}

Ainsi, cette analyse des effets génétiques sur la croissance pondérale relatifs aux races Charolaise et Aberdeen Angus a conduit à mettre en évidence les phénomènes suivants :

Tout d'abord, le poids de naissance plus élevé des veaux Charolais par rapport à ceux de race Aberdeen Angus résulte avant tout d'une supériorité d'effets génétiques additifs directs et maternels de la race Charolaise pour ce caractère. En plus de ces effets additifs, il apparaît en croisement entre ces deux races des effets d'hétérosis direct relativement importants.

Durant la phase d'allaitement, les veaux comportant des gènes Charolais manifestent une croissance journalière plus élevée du fait d'effets génétiques directs plus importants qui, joints à la supériorité de leurs effets additifs directs sur le poids à la naissance, leur confèrent un poids au sevrage plus élevé que les veaux comportant des gènes Aberdeen Angus. Cependant, relativement à leur poids, les veaux croisés Aberdeen Angus croissent plus rapidement ce qui explique que les différences d'effets additifs directs entre ces deux races soient moins importants au sevrage qu'à la naissance. De plus ce phénomène est accentué par le fait que durant cette phase avant sevrage, il n'apparaît pas de supériorité de la race Charolaise pour les effets génétiques additifs maternels sur la croissance; au contraire, la race Aberdeen Angus apparaîtrait plutôt supérieure sur la croissance relative.

Par ailleurs, les valeurs estimées des effets d'hétérosis entre ces deux races laissent supposer que, dans nos conditions d'élevage des animaux, l'incidence de l'hétérosis sur les effets directs (hétérosis direct) serait plus importante sur la croissance prénatale et après le sevrage alors que l'incidence sur les effets maternels (hétérosis maternel) serait prépondérante sur la croissance entre la naissance et le sevrage.

Enfin, sur les caractères de croissance pondérale il s'avère que les effets dus aux pertes épistatiques de recombinaisons sont peu importants du moins entre la race Charolaise et Aberdeen Angus.

Finalement, l'ensemble de ces estimations des différentes composantes génétiques pour la croissance absolue et relative aux divers stades de la vie, permettent maintenant de rechercher efficacement l'utilisation optimale du croisement entre ces deux races à viande de taille différente ; ceci est particulièrement important pour les 
génisses vêlant précocement où il est nécessaire de trouver un optimum entre la croissance pré-natale et la croissance post-natale pour contrôler les risques de difficultés de vêlage liées à l'excès de taille du veau par rapport à celle de sa mère.

Reçu pour publication en janvier 1982.

\section{Remerciements}

Les auteurs tiennent à remercier MM. F. Menissier et L. Santa Coloma pour leur collaboration à la traduction de ce texte, MM. R. Sullivan et R. Sofiack pour leur assistance technique dans cette expérimentation, ainsi que M. J.L. Foulley pour ses pertinentes critiques et suggestions lors de l'analyse de ces données.

\section{Summary}

Genetic components of growth parameters computed in a crossbreeding experiment between Aberdeen Angus and Charolais breeds

Individual and maternal heterosis, differences in average breed transmitted and maternal effects and changes in inter-allelic gene interaction effects were estimated for the Angus and Charolais breeds. The traits analyzed included birth, weaning and 20 month weight, and absolute and relative daily gain among these periods. Data from 815 and 605 animals born over 1966-1971 period at the "Estación Experimental Agropecuaria - I.N.T.A.» of Balcarce (Argentina) were analyzed for pre- and post-weaning performance respectively. Angus, Charolais, F1, F2 and backcrossed animals were utilized to estimate the genetic parameters. Charolais individual average effects were significantly higher than Angu's for all traits. The maternal ability of the Charolais was higher than Angu's only for birth weight. Individual heterosis was $+11 \%$ and $+12 \%$ for birth weight and weight at 20 months respectively. Maternal heterosis was not statistically significant for any trait but reached values of approximately $+7 \%$ for the preweaning period. The change is non allelic gene interactions due to chromosomic recombinations was not important for any traits.

\section{Références bibliographiques}

Cabrini E.J., Cavandoli H.H., 1964. Cruzas con Charolais en Marcos Juarez. Bol. tec. de I.N.TA., $\mathrm{n}^{\circ} 19,34 \mathrm{p}$.

Cundiff L.V., Gregory K.E., Schwulst F.J., Koch R.M., 1974. Effects of heterosis on maternal performance and milk production in Hereford, Angus and Shorthorn cattle. J. anim. Sci., 38, 728-745.

Departamento de producción animal, 1974. Resultados de cruzamientos con bovinos para carne. I.N.T.A., Est. Exper. Reg. Agrop. de Balcarce. Materiales didacticos, $\mathrm{n}^{\circ} \mathbf{3}$, $74 \mathrm{p}$.

Dickerson G., 1969. Experimental approaches in utilizing breed resources. Anim. Breed. Abstr., 37, 191-202. 
Dickerson G., 1972. Inbreeding and heterosis in animals. In : Proc. Anim. Breed. Genet. Symp., Blasksburg, Virginia, pp. 54-77. Am. Soc. anim. Sci. and Am. Dairy Sci. Assoc.

Foulley J.L., LeFort G., 1978. Méthodes d'estimation des effets directs et maternels en sélection animale. Ann. Génét. Sél. anim., 10, 475-496.

Gregory K.E., Cundiff L.V., Koch R.M., Laster D.B., Smith G.M., 1978. Heterosis and breed maternal and transmitted effects in beef cattle. I - Preweaning traits. J. anim. Sci., 47, 1031-1041.

Jain J.P., Lasley J.F., Sibitt B., LangFord L., Comport J.E., Dyer A.J., Krause G.F., HEDRICK H.B., 1971. Growth traits of reciprocally crossed Angus, Hereford and Charolais heifers. J. anim. Sci., 32, 399-405.

lasley L.F., Sibbit B., Langford L., Comport J.B., Dyer A.J., Krause G.E., Hedrick H.B., 1973. Growth traits in straightbreed and reciprocally crossed Angus, Hereford and Charolais steers. J. anim. Sci., 36, 1044-1056.

lopez Saubidet C., Cavandoli H., Igartua O.A., Joandet G.E., Cabrini E.J., Villlard J.A., Sivori I.H., Hernandez O., Covas G., Kugler W.F., 1963. Cruzas con Charolais en la región pampeana. I.N.T.A., Est. Exp. Agrop. de Balcarce. Bol. Tec., $\mathrm{n}^{0}$ 6, $74 \mathrm{p}$.

Miquel M.C., Molinuevo H.A., Joandet G.E., Lopez Saubidet C., Bidart J.B., 1974 a. Evaluación de crecimiento hasta el destete de cruzamientos de razas no tracionales con vientres Aberdeen Angus. Prod. anim. (Argentina), 5, 143-159.

Miquel M.C., Molinuevo H.A., Joandet G.E., Lopez Saubidet C., Bidart J.B., 1974 b. Evaluación de crecimiento en invernada de cruzamientos de razas no tradicionales con vientres Aberdeen Angus. Prod. anim. (Argentina), 5, 160-169.

Molinuevo H.A., 1967. Estimación del peso al destete por unidad de superficie y su relación con el tamano de las vacas. R.I.A., I.N.T.A. (Argentina), 4, 37-47.

Molinuevo H., 1970. Effets d'hétérosis sur les poids à la naissance et au sevrage dans le croisement Charolais et Aberdeen Angus. Ann. Génét. Sél. anim., 2, 75-84.

Molinuevo H., Joandet G.E., Bidart J.B., 1974. Comportamiento reproductivo de animales Aberdeen Angus, Charolais y sus cruzas. Prod. anim. (Argentina), 3, 197-208.

Peacock F.M., Koger M., Hodges E.M., 1978. Weaning traits of Angus, Brahman, Charolais and $\mathrm{F} 1$ crosses of these breeds. J. anim. Sci., 47, 366-369.

Olson L.W., CundifF L.V., Gregory K.E., 1978. Maternal heterosis effects on postweaning growth and carcass traits in beef cattle. J. anim. Sci., 46, 1552-1562.

Sagebiel J.A., Sibbit W.R., Langford L.L., Dyer A.J., Comport J.E., Lasley J.F., 1967. Heterosis in weaning, post weaning and slaughter traits of beef heifers. J. anim. Sci., 26, 889 (Abstr.).

SeArLe S.R., 1974. Linear Models. John Wiley y Sons, Inc. New York, London, Sydney, Toronto, $532 \mathrm{p}$. 